\title{
Massive scalar field in multiply connected flat spacetimes
}

\author{
Tsunefumi Tanakat and William A. Hiscock \\ Department of Physics, Montana State University, Bozeman, Montana 59717
}

(October 27, 2018)

\begin{abstract}
The vacuum expectation value of the stress-energy tensor $\left\langle 0\left|T_{\mu \nu}\right| 0\right\rangle$ is calculated in several multiply connected flat spacetimes for a massive scalar field with arbitrary curvature coupling. We find that a nonzero field mass always decreases the magnitude of the energy density in chronology-respecting manifolds such as $R^{3} \times S^{1}, R^{2} \times T^{2}, R^{1} \times T^{3}$, the Möbius strip, and the Klein bottle. In Grant space, which contains nonchronal regions, whether $\left\langle 0\left|T_{\mu \nu}\right| 0\right\rangle$ diverges on a chronology horizon or not depends on the field mass. For a sufficiently large mass $\left\langle 0\left|T_{\mu \nu}\right| 0\right\rangle$ remains finite, and the metric backreaction caused by a massive quantized field may not be large enough to significantly change the Grant space geometry.
\end{abstract}

Typeset using REVTEX 


\section{INTRODUCTION}

The global topology of spacetime, which is not fixed by the equations of general relativity, plays an important role in quantum field theory even in a flat spacetime. When a spacetime is multiply connected only those modes of a field that satisfy boundary conditions determined by the topology of the spacetime are relevant in the calculation of locally measurable quantities such as the stress-energy tensor $T_{\mu \nu}$. For example, in a cylindrical two dimensional spacetime, $R^{1}$ (time) $\times S^{1}$ (space), the only allowed momentum is an integer multiple of $\frac{2 \pi \hbar}{a}$ where $a$ is the circumference in the closed spatial direction. DeWitt, Hart, and Isham [1] thoroughly studied the effects of multiple connectedness of the spacetime manifold (called Möbiosity), twisting of the field, and orientability of manifold on $\left\langle 0\left|T_{\mu \nu}\right| 0\right\rangle$ for a massless scalar field in various topological spaces. In this paper we extend their work to an untwisted massive scalar field. We evaluate $\left\langle 0\left|T_{\mu \nu}\right| 0\right\rangle$ in four dimensional spacetime manifolds of the type $R^{1}$ (time) $\times \Sigma\left(\right.$ space $\left.^{3}\right)$ where $\Sigma$ can be either $S^{1} \times R^{2}, T^{2} \times R^{1}, T^{3}$, the Möbius strip $M^{2} \times R^{1}$, or the Klein bottle $K^{2} \times R^{1}$. Both the Möbius strip and the Klein bottle are examples of nonorientable manifolds.

Another issue addressed in this paper is the effect of field mass on chronology protection. In previous papers [2-10] it has been shown that $\left\langle 0\left|T_{\mu \nu}\right| 0\right\rangle$ for a massless scalar field diverges on a chronology horizon in various spacetimes with closed timelike curves (CTC's). The backreaction of the metric to this diverging stress-energy through the Einstein field equations may be able to prevent formation of CTC's. However, Boulware's work indicates that $\left\langle 0\left|T_{\mu \nu}\right| 0\right\rangle$ for a massive scalar field remains finite on the chronology horizon in Gott space [6]. We have confirmed Boulware's result in Grant space which is holonomic to Gott space [10] and found that $\left\langle 0\left|T_{\mu \nu}\right| 0\right\rangle$ is finite on the chronology horizon provided the mass of the scalar field is above a lower limit which depends on the topological identification scale lengths of the spacetime.

In Sec. II a general procedure for using point-splitting regularization to calculate the vacuum expectation value of $T_{\mu \nu}$ for a free massive scalar field in a flat but multiply connected 
spacetime is described. We then apply this method to spacetimes without CTC's in Sec. III and to those with CTC's in Sec. IV. Throughout our calculations natural units in which $c=G=\hbar=1$ are used and the metric signature is +2 .

\section{CALCULATION OF $\left\langle 0\left|T_{\mu \nu}\right| 0\right\rangle$ IN A MULTIPLY CONNECTED FLAT SPACETIME}

Our calculation of the vacuum expectation value of the stress-energy tensor is greatly simplified by the facts that all curvature components vanish in a flat spacetime and that we do not need to deal with renormalization of the field mass $M$ and curvature coupling constant $\xi$ arising from interactions. However, we do have to worry about the topology of the spacetime manifold since it may allow two points on the manifold to be connected by multiple geodesics. In this paper the Minkowski vacuum state is assumed to be the default vacuum state in all spacetimes considered. This assumption is defended later by an argument based on a particle detector carried by a geodesic observer. Point-splitting regularization (or the "method of images") is used to take multiple connectedness into account. The differences in topology of the spacetimes will appear only in the geodesic distances between image charges and in the number of geodesics connecting the points. Once geodesical distances for a particular topology are found the calculation of $\left\langle 0\left|T_{\mu \nu}\right| 0\right\rangle$ reduces to simple differentiation of the Hadamard elementary function and taking the coincidence limit.

The stress-energy tensor $T_{\mu \nu}$ is formally defined as a variation of the action with respect to the metric. In a flat four dimensional spacetime the stress-energy tensor for a general free scalar field is given by

$$
\begin{aligned}
T_{\mu \nu}= & (1-2 \xi) \phi_{; \mu} \phi_{; \nu}+\left(2 \xi-\frac{1}{2}\right) g_{\mu \nu} \phi_{; \alpha} \phi^{; \alpha}-2 \xi \phi \phi_{; \mu \nu} \\
& +2 \xi g_{\mu \nu} \phi \square \phi-\frac{1}{2} M^{2} g_{\mu \nu} \phi^{2} .
\end{aligned}
$$

Note that $\left\langle 0\left|T_{\mu \nu}\right| 0\right\rangle$ depends upon the value of $\xi$ even when the curvature vanishes. For conformal coupling $\xi=\frac{1}{6}$; for minimal coupling $\xi=0$. We will allow arbitrary values of 
$\xi$ to make our results as general as possible. The scalar field $\phi$ satisfies the Klein-Gordon equation $\left(\square_{x}-M^{2}\right) \phi(x)=0$.

Since every term in $T_{\mu \nu}$ is quadratic in the field variable $\phi(x)$, we can split the point $x$ into $x$ and $\tilde{x}$ and take the coincidence limit as $\tilde{x} \rightarrow x$.

$$
\begin{aligned}
T_{\mu \nu}= & \frac{1}{2} \lim _{\tilde{x} \rightarrow x}\left[(1-2 \xi) \nabla_{\mu} \widetilde{\nabla}_{\nu}+\left(2 \xi-\frac{1}{2}\right) g_{\mu \nu} \nabla_{\alpha} \widetilde{\nabla}^{\alpha}-2 \xi \nabla_{\mu} \nabla_{\nu}\right. \\
& \left.+2 \xi g_{\mu \nu} \nabla_{\alpha} \nabla^{\alpha}-\frac{1}{2} M^{2} g_{\mu \nu}\right]\{\phi(x), \phi(\tilde{x})\}
\end{aligned}
$$

where $\{A, B\}$ is the anticommutator of $A$ and $B$. Covariant derivatives $\nabla_{\mu}$ and $\widetilde{\nabla}_{\nu}$ are to be applied with respect to $x$ and $\tilde{x}$. We have also symmetrized $T_{\mu \nu}$ over $\phi(x)$ and $\phi(\tilde{x})$. Before taking the vacuum expectation value of $T_{\mu \nu}$ we need to define the vacuum state of the spacetimes. This is nontrivial because some spacetimes lack a global timelike Killing vector field, which is required to define positive frequency.

The spacetimes we will consider may all be constructed by making topological identifications of Minkowski space, with coordinates and metric given by

$$
d s^{2}=-\left(d x^{0}\right)^{2}+\left(d x^{1}\right)^{2}+\left(d x^{2}\right)^{2}+\left(d x^{3}\right)^{2} .
$$

In the case of the $R^{3} \times S^{1}, R^{2} \times T^{2}, R^{1} \times T^{3}$, the Möbius strip and the Klein bottle spacetimes, there exists a global timelike Killing vector field (i. e., $\frac{\partial}{\partial x^{0}}$ ) and so does a natural vacuum state. The effect of multiple connectedness on the field is only making it periodic in closed spatial directions. Also, it allows the field to be twisted, and we will briefly comment of the effect of twisting and the vacuum energy density in the next section. Since each section of these spacetimes between the boundaries is identical to a portion of Minkowski space, we can assume the default vacuum states of these spacetimes to be identical to that of ordinary Minkowski space.

As an example of a spacetime with no natural vacuum state, we will consider Grant space in Sec. IV. Although Grant space does have local timelike Killing vector fields everywhere, they can not be patched together to form a global timelike Killing vector field [2]. However, each section of the spacetime is identical to Minkowski space, and a geodesic observer will 
not detect any particles if the spacetime is in the Minkowski vacuum state. Hence, we can also use the Minkowski vacuum state for the Grant space.

The vacuum expectation value of stress-energy tensor is now given by sandwiching both sides of Eq. (2) by the Minkowski vacuum state $|0\rangle$.

$$
\begin{aligned}
\left\langle 0\left|T_{\mu \nu}\right| 0\right\rangle= & \frac{1}{2} \lim _{\tilde{x} \rightarrow x}\left[(1-2 \xi) \nabla_{\mu} \widetilde{\nabla}_{\nu}+\left(2 \xi-\frac{1}{2}\right) g_{\mu \nu} \nabla_{\alpha} \widetilde{\nabla}^{\alpha}-2 \xi \nabla_{\mu} \nabla_{\nu}\right. \\
& \left.+2 \xi g_{\mu \nu} \nabla_{\alpha} \nabla^{\alpha}-\frac{1}{2} M^{2} g_{\mu \nu}\right] G^{(1)}(x, \tilde{x})
\end{aligned}
$$

where the Hadamard elementary function $G^{(1)}(x, \tilde{x})$ is defined as

$$
G^{(1)}(x, \tilde{x})=\langle 0|\{\phi(x), \phi(\tilde{x})\}| 0\rangle
$$

and satisfies the Klein-Gordon equation $\left(\square_{x}-M^{2}\right) G^{(1)}(x, \tilde{x})=0$. In Minkowski space $G^{(1)}(x, \tilde{x})$ is a function of the half squared geodesic distance $\sigma=\frac{1}{2} g_{\alpha \beta}\left(x^{\alpha}-\tilde{x}^{\alpha}\right)\left(x^{\beta}-\tilde{x}^{\beta}\right)$ between two points $x$ and $\tilde{x}$ and has the form

$$
\begin{aligned}
G^{(1)}(x, \tilde{x})= & \frac{M}{2 \pi^{2} \sqrt{2 \sigma}} \Theta(2 \sigma) K_{1}(M \sqrt{2 \sigma}) \\
& +\frac{M}{4 \pi \sqrt{-2 \sigma}} \Theta(-2 \sigma) I_{1}(M \sqrt{-2 \sigma}),
\end{aligned}
$$

where $\Theta$ is a step function and $I_{1}$ and $K_{1}$ are modified Bessel functions of the first and second kinds, respectively [12].

Because the spacetime is multiply connected, there can be more than one geodesic connecting the two points $x$ and $\tilde{x}$. For example, suppose the spacetime is closed in the $x^{1}$ direction. We can connect $x$ and $\tilde{x}$ with a direct path, or we can start from $x$ and circle around in the $x^{1}$ direction once, twice, or an arbitrary number of times before arriving at $\tilde{x}$. Since the path circling around $n$ times cannot be deformed continuously into the one which circles around $n^{\prime}\left(n^{\prime} \neq n\right)$ times, all inequivalent paths must be taken into account. Equivalently we can consider this situation as an electrostatic problem and use the method of images. The "image charges" of the point $\tilde{x}$ are located at $\tilde{x} \pm a, \tilde{x} \pm 2 a, \cdots, \tilde{x} \pm n a$, where $a$ is the periodicity (or circumference) in the closed spatial direction. All these image 
charges are connected to the point $x$ by geodesics whose half squared distances $\sigma_{n}$ are given by

$$
\sigma_{n}=\frac{1}{2} g_{\alpha \beta}\left(x^{\alpha}-\tilde{x}_{n}^{\alpha}\right)\left(x^{\beta}-\tilde{x}_{n}^{\beta}\right)
$$

where $\tilde{x}_{n}$ is the position of the $n$th image charge. A contribution from each image charge is summed over to construct the regularized Hadamard function $G_{\mathrm{reg}}^{(1)}$. However, using $G_{\mathrm{reg}}^{(1)}$ in Eq. (4) gives infinity because the stress-energy tensor is not renormalized yet. The infinite vacuum energy term associated with the Minkowski vacuum state must be subtracted from $G_{\text {reg }}^{(1)}$. This term comes from $G^{(1)}$ for the image charge at $\tilde{x}_{0}$. Excluding it from the summation we obtain the renormalized Hadamard function $G_{\text {ren }}^{(1)}$ which, using Eq. (đ), gives the renormalized stress-energy tensor $\left\langle 0\left|T_{\mu \nu}\right| 0\right\rangle_{\text {ren }}$.

$$
G_{\mathrm{ren}}^{(1)}(x, \tilde{x})=\sum_{\substack{n=-\infty \\ n \neq 0}}^{\infty} G^{(1)}\left(\sigma_{n}\right)
$$

The calculation of $\left\langle 0\left|T_{\mu \nu}\right| 0\right\rangle$ has thus been reduced to (1) writing an appropriate $\sigma_{n}$ for each topology, (2) applying the derivative operator in Eq. (4), and (3) taking the coincidence limit as $\tilde{x} \rightarrow x$.

\section{SPACETIMES WITH A GLOBAL TIMELIKE KILLING VECTOR FIELD}

\section{A. Orientable manifolds}

In this section the vacuum expectation value $\left\langle 0\left|T_{\mu \nu}\right| 0\right\rangle$ of the stress-energy tensor of a massive scalar field is evaluated in four dimensional spacetimes with $R($ time $) \times \Sigma\left(\right.$ space $\left.^{3}\right)$ topology. A spacetime of this type has a global timelike Killing vector field (e. g., $\frac{\partial}{\partial x^{0}}$ ) and thus a natural vacuum state. The first topology we investigate is $\Sigma=S^{1} \times R^{2}$. The manifold can be either orientable or nonorientable. In an orientable manifold a triad obeying the right-hand rule maintains the same handedness as it is translated around the closed spatial dimension. In Cartesian coordinates $\left(x^{0}, x^{1}, x^{2}, x^{3}\right)$ the spacetime is closed in the $x^{1}$ direction with periodicity $a$, and the following points are identified: 


$$
\left(x^{0}, x^{1}, x^{2}, x^{3}\right) \leftrightarrow\left(x^{0}, x^{1}+n a, x^{2}, x^{3}\right)
$$

where $n$ is an integer. The half squared geodesic distance $\sigma_{n}$ between the point $x$ and the $n$th image charge at $\tilde{x}_{n}$ is equal to

$$
\sigma_{n}=\frac{1}{2}\left[-\left(x^{0}-\tilde{x}^{0}\right)^{2}+\left(x^{1}-\tilde{x}^{1}-n a\right)^{2}+\left(x^{2}-\tilde{x}^{2}\right)^{2}+\left(x^{3}-\tilde{x}^{3}\right)^{2}\right] .
$$

We will be concerned with only a spacelike separation $\left(\sigma_{n}>0\right)$, so the first term in Eq. (6) is used for the Hadamard function. This is because the intervals between the image charges are always spacelike in all spacetimes concerned.

Using the prescription for the calculation of $\left\langle 0\left|T_{\mu \nu}\right| 0\right\rangle$ described in the previous section, we obtain

$$
\left\langle 0\left|T_{\mu \nu}\right| 0\right\rangle=\frac{M^{4}}{2 \pi^{2}} \sum_{n=1}^{\infty}\left\{\frac{K_{2}\left(z_{n}\right)}{z_{n}^{2}} g_{\mu \nu}+\frac{K_{3}\left(z_{n}\right)}{z_{n}} \operatorname{diag}[0,-1,0,0]\right\},
$$

where $z_{n}=$ Mna. $\left\langle 0\left|T_{\mu \nu}\right| 0\right\rangle$ does not depend on the curvature coupling. The energy density $\rho=\left\langle 0\left|T_{00}\right| 0\right\rangle$ is negative due to a fact that only certain wavelengths are allowed in the $x^{1}$ direction compared to Minkowski space in which all wavelengths are allowed. In the massless limit the above result reduces to that given in Ref. [1]. Figure 1 shows a plot of $\rho$ vs. $M$ for $a=1$. The effect of mass on the energy density is to raise it (decreasing its magnitude) because for a larger field mass, its characteristic wavelength or Compton wavelength $\lambda_{C}=\frac{1}{M}$ becomes shorter and is less sensitive to the global structure of the manifold.

The next manifold that we will consider is $\Sigma=T^{2} \times R^{1}$. The spacetime is now closed in two spatial dimensions, $x^{1}$ and $x^{2}$, with periodicities $a$ and $b$, respectively. The following points are identified

$$
\left(x^{0}, x^{1}, x^{2}, x^{3}\right) \leftrightarrow\left(x^{0}, x^{1}+n a, x^{2}+m b, x^{3}\right)
$$

where $n$ and $m$ are integers. The half squared geodesic distance $\sigma_{n m}$ between $x$ and the $n m$ th image charge at $\tilde{x}_{n m}$ is given by

$$
\sigma_{n m}=\frac{1}{2}\left[-\left(x^{0}-\tilde{x}^{0}\right)^{2}+\left(x^{1}-\tilde{x}^{1}-n a\right)^{2}+\left(x^{2}-\tilde{x}^{2}-m b\right)^{2}+\left(x^{3}-\tilde{x}^{3}\right)^{2}\right] .
$$


Following the prescription we find

$$
\begin{aligned}
\left\langle 0\left|T_{\mu \nu}\right| 0\right\rangle= & \frac{M^{4}}{4 \pi^{2}} \sum_{\substack{n, m=-\infty \\
(n, m) \neq(0,0)}}^{\infty}\left\{\frac{K_{2}\left(z_{n m}\right)}{z_{n m}^{2}} g_{\mu \nu}\right. \\
& \left.+M^{2} \frac{K_{3}\left(z_{n m}\right)}{z_{n m}^{3}} \operatorname{diag}\left[0,-n^{2} a^{2},-m^{2} b^{2}, 0\right]\right\},
\end{aligned}
$$

where $z_{n m}=M\left(n^{2} a^{2}+m^{2} b^{2}\right)^{\frac{1}{2}}$. The summation is over all values of $n$ and $m$ except when $n=m=0$ simultaneously. As it can be seen in Fig. 1 $\rho$ for $R^{2} \times T^{2}$ is lower (greater in magnitude) than that for $R^{3} \times T^{1}$. The increased Möbiosity of the manifold lowers the energy density [1].

The last example of orientable spacetime manifold is the one which is closed in all spatial directions $\left(\Sigma=T^{3}\right)$. The topology of this spacetime is such that the following points are identified:

$$
\left(x^{0}, x^{1}, x^{2}, x^{3}\right) \leftrightarrow\left(x^{0}, x^{1}+n a, x^{2}+m b, x^{3}+l c\right)
$$

where $n, m$, and $l$ are integers and $a, b$, and $c$ are the periodicities in the $x^{1}, x^{2}$, and $x^{3}$ directions, respectively. The half squared geodesic distance $\sigma_{n m l}$ is equal to

$$
\begin{aligned}
\sigma_{n m l}= & \frac{1}{2}\left[-\left(x^{0}-\tilde{x}^{0}\right)^{2}+\left(x^{1}-\tilde{x}^{1}-n a\right)^{2}+\left(x^{2}-\tilde{x}^{2}-m b\right)^{2}\right. \\
& \left.+\left(x^{3}-\tilde{x}^{3}-l c\right)^{2}\right]
\end{aligned}
$$

and the resulting stress-energy tensor is

$$
\begin{aligned}
\left\langle 0\left|T_{\mu \nu}\right| 0\right\rangle= & \frac{M^{4}}{4 \pi^{2}} \sum_{\substack{n, m, l=-\infty \\
(n, m, l) \neq(0,0,0)}}^{\infty}\left\{\frac{K_{2}\left(z_{n m l}\right)}{z_{n m l}^{2}} g_{\mu \nu}\right. \\
& \left.+M^{2} \frac{K_{3}\left(z_{n m l}\right)}{z_{n m l}^{3}} \operatorname{diag}\left[0,-n^{2} a^{2},-m^{2} b^{2},-l^{2} c^{2}\right]\right\},
\end{aligned}
$$

where $z_{n m l}=M\left(n^{2} a^{2}+m^{2} b^{2}+l^{2} c^{2}\right)^{\frac{1}{2}}$. The summation is over all possible value of $n, m$, and $l$ except when $n=m=l=0$ at the same time. As we can see in Fig. 1 the increased Möbiosity lowers the vacuum energy density $\rho$. Increasing the field mass raises it toward zero. 
The procedure described previous section is readily applicable to the twisted field. The only modification required is to introduce the factor of $(-1)^{n}$ in the summation in Eq. (8)). This will make field antiperiodic. DeWitt, Hart, and Isham have shown that twisting of the field raises $\rho$ above the zero-value of Minkowski vacuum state for a massless scalar field [1]. Our numerical calculations indicate that this is true even for a massive scalar field.

\section{B. Nonorientable manifolds}

The manifolds we have examined so far are orientable. Next, we examine how the orientation of the manifold affects the vacuum polarization. In a nonorientable manifold a triad which defines the right-hand rule flips to the one for the left-hand rule when it is transported around the closed spatial dimension. The simplest example of a nonorientable manifold is a Möbius strip $\left(M^{2}\right)$, which locally looks like the $R^{1} \times S^{1}$ manifold [13]. A four dimensional Möbius strip spacetime $R$ (time) $\times M^{2} \times R$ (space) can be constructed by identifying the points

$$
\left(x^{0}, x^{1}, x^{2}, x^{3}\right) \leftrightarrow\left(x^{0}, x^{1}+n a,(-1)^{n} x^{2}, x^{3}\right)
$$

The half squared geodesic distance $\sigma_{n}$ is equal to

$$
\sigma_{n}=\frac{1}{2}\left\{-\left(x^{0}-\tilde{x}^{0}\right)^{2}+\left(x^{1}-\tilde{x}^{1}-n a\right)^{2}+\left[x^{2}-(-1)^{n} \tilde{x}^{2}\right]^{2}+\left(x^{3}-\tilde{x}^{3}\right)^{2}\right\} .
$$

It is obvious that $\sigma_{n}$ and thus $\left\langle 0\left|T_{\mu \nu}\right| 0\right\rangle$ will depend on $x^{2}$ even after the coincidence limit is taken.

$$
\begin{aligned}
\left\langle 0\left|T_{\mu \nu}\right| 0\right\rangle= & \frac{M^{4}}{2 \pi^{2}} \sum_{n=1}^{\infty}\left\{\frac{K_{2}\left(z_{2 n}\right)}{\left(z_{2 n}\right)^{2}} g_{\mu \nu}+\frac{K_{3}\left(z_{2 n}\right)}{z_{2 n}} \operatorname{diag}[0,-1,0,0]\right\} \\
& +\frac{M^{4}}{4 \pi^{2}} \sum_{n=-\infty}^{\infty}\left\{\left[2(1-2 \xi) \frac{K_{2}\left(z_{2 n+1}\right)}{\left(z_{2 n+1}\right)^{2}}+4(4 \xi-1) M^{2}\left(x^{2}\right)^{2} \frac{K_{3}\left(z_{2 n+1}\right)}{\left(z_{2 n+1}\right)^{3}}\right]\right. \\
& \times \operatorname{diag}[-1,1,0,1] \\
& \left.+M^{2}(2 n+1)^{2} a^{2} \frac{K_{3}\left(z_{2 n+1}\right)}{\left(z_{2 n+1}\right)^{3}} \operatorname{diag}[0,-1,0,0]\right\},
\end{aligned}
$$

where $z_{2 n}=2 M n a$ and $z_{2 n+1}=M\left[(2 n+1)^{2} a^{2}+4\left(x^{2}\right)^{2}\right]^{\frac{1}{2}}$. The first term is identical to that for the case of $R^{3} \times S^{1}$ with twice the periodicity. The periodicity is doubled because the 
triad must circle around in the $x^{1}$ direction twice in order to be oriented in the original sense. It is interesting that the second term in Eq. (20) is dependent on the curvature coupling constant $\xi ; \rho$ increases linearly with increasing $\xi$. For the Möbius strip the nonorientability of the manifold lowers the energy density from that of $R^{3} \times S^{1}$ as we can see in Fig. 2. Along the $x^{2}$ direction $\rho$ has a reverse bell shape centered at $x^{2}=0$. A plot of $\rho$ vs. $x^{2}$ is shown in Fig. 3 .

The last example of a spacetime with $R^{1} \times \Sigma$ topology is the Klein bottle spacetime $\left(\Sigma=K^{2} \times R^{1}\right)$. The Klein bottle $K^{2}$ is a natural extension of the Möbius strip with an additional spatial periodic boundary condition. In Cartesian coordinates, the points to be identified are

$$
\left(x^{0}, x^{1}, x^{2}, x^{3}\right) \leftrightarrow\left(x^{0}, x^{1}+n a,(-1)^{n} x^{2}+2 m b, x^{3}\right)
$$

and $\sigma_{n m}$ is equal to

$$
\begin{aligned}
\sigma_{n m}= & \frac{1}{2}\left[-\left(x^{0}-\tilde{x}^{0}\right)^{2}+\left(x^{1}-\tilde{x}^{1}-n a\right)^{2}+\left\{x^{2}-(-1)^{n} \tilde{x}^{2}-2 m b\right\}^{2}\right. \\
& \left.+\left(x^{3}-\tilde{x}^{3}\right)^{2}\right] .
\end{aligned}
$$

Again, the resulting $\left\langle 0\left|T_{\mu \nu}\right| 0\right\rangle$ depends on both $x^{2}$ and $\xi$,

$$
\begin{aligned}
\left\langle 0\left|T_{\mu \nu}\right| 0\right\rangle= & \frac{M^{4}}{4 \pi^{2}} \sum_{\substack{n, m=-\infty \\
(n, m) \neq(0,0)}}^{\infty}\left\{\frac{K_{2}\left(z_{2 n, m}\right)}{\left(z_{2 n, m}\right)^{2}} g_{\mu \nu}+M^{2} \frac{K_{3}\left(z_{2 n, m}\right)}{\left(z_{2 n, m}^{3}\right)}\right. \\
& \left.\times \operatorname{diag}\left[0,-(2 n)^{2} a^{2},-(2 m)^{2} b^{2}, 0\right]\right\} \\
& +\frac{M^{4}}{4 \pi^{2}} \sum_{n, m=-\infty}^{\infty}\left\{\left[2(1-2 \xi) \frac{K_{2}\left(z_{2 n+1, m}\right)}{\left(z_{2 n+1, m}\right)^{2}}\right.\right. \\
& \left.+4(4 \xi-1) M^{2}\left(x^{2}-m b\right)^{2} \frac{K_{3}\left(z_{2 n+1, m}\right)}{\left(z_{2 n+1, m}\right)^{3}}\right] \operatorname{diag}[-1,1,0,1] \\
& \left.+M^{2}(2 n+1)^{2} a^{2} \frac{K_{3}\left(z_{2 n+1, m}\right)}{\left(z_{2 n+1, m}\right)^{3}} \operatorname{diag}[0,-1,0,0]\right\},
\end{aligned}
$$

where $z_{2 n, m}=2 M\left(n^{2} a^{2}+m^{2} b^{2}\right)^{\frac{1}{2}}$ and $z_{2 n+1, m}=M\left[(2 n+1)^{2} a^{2}+4\left(x^{2}-m b\right)^{2}\right]^{\frac{1}{2}}$. The first summation is identical to $\left\langle 0\left|T_{\mu \nu}\right| 0\right\rangle$ for $R^{2} \times T^{2}$ with spatial periodicities doubled in both $x^{1}$ and $x^{2}$ directions. The first summation simply gives a constant negative shift in the 
vacuum stress-energy throughout the spacetime. The second half of $\left\langle 0\left|T_{\mu \nu}\right| 0\right\rangle$ is dependent on both the $x^{2}$ coordinate and the curvature coupling $\xi . \rho$ is oscillatory in the $x^{2}$ direction with minima at integer multiples of the periodicity $b$ in that direction (See Fig. 3). As in the Möbius strip spacetime an increase in the value of $\xi$ causes $\rho$ to increase. However, in contrast to the Möbius strip spacetime, the nonorientability of the manifold decreases the magnitude of the energy density compared to the $R^{2} \times T^{2}$ spacetime as we can see in Fig. 2.

\section{A SPACETIME WITHOUT A GLOBAL TIMELIKE KILLING VECTOR FIELD}

The spacetimes that we have examined so far have one thing in common; they have a clearly defined Killing time coordinate $x^{0}$. In those spacetimes $\frac{\partial}{\partial x^{0}}$ is a globally defined timelike Killing vector field which can be used to define a vacuum state. However, it is possible to arrange topological identification of flat space such that a clearly defined time coordinate for the entire spacetime manifold does not exist. As an example of a spacetime without a global timelike Killing vector field, we will consider Grant space.

Grant space is interesting because it contains closed timelike curves (CTC's). The chronology protection conjecture, proposed by Hawking [14, states that nature prevents the formation of CTC's in spacetimes which have an initial chronal region. In order to prove the conjecture, the protection mechanism within the laws of physics must be identified, if it exists. Currently the most promising candidate for the protection mechanism is the backreaction on the metric due to vacuum polarization of quantized matter fields. Quantum field fluctuations pile up on top of themselves near a chronology horizon causing the vacuum expectation value of stress-energy tensor $\left\langle 0\left|T_{\mu \nu}\right| 0\right\rangle$ to grow without bound. This diverging $\left\langle 0\left|T_{\mu \nu}\right| 0\right\rangle$ will act back on the metric through the Einstein field equations and change the spacetime geometry, possibly preventing the appearance of CTC's. The vacuum stress-energy of a massless scalar field has been shown to diverge on the chronology horizon

in Misner space [2], Gott space [6], wormhole spacetime [4], and Roman space [8]. In our 
previous paper [9] we evaluated $\left\langle 0\left|T_{\mu \nu}\right| 0\right\rangle$ for a massive scalar field with arbitrary curvature coupling in Misner space and found that it diverges on the chronology horizon. On the other hand, Boulware [6] has shown that the vacuum stress-energy of a massive scalar field is finite on the chronology horizon of Gott space. We would like to know whether that is still true in the Grant space, which is holonomic to Gott space yet contains Misner space as a special limit.

The original Misner space was developed to illustrate topological pathologies associated with Taub-NUT (Newman-Unti-Tamburino) type spacetimes [15,16]. Misner space is simply the flat Kasner universe with an altered topology. Its metric in Misner coordinates $\left(y^{0}, y^{1}, y^{2}, y^{3}\right)$ is

$$
d s^{2}=-\left(d y^{0}\right)^{2}+\left(y^{0}\right)^{2}\left(d y^{1}\right)^{2}+\left(d y^{2}\right)^{2}+\left(d y^{3}\right)^{2} .
$$

That Misner space is flat can be easily seen; the above metric becomes identical to the Minkowski metric via the following coordinate transformation:

$$
x^{0}=y^{0} \cosh y^{1}, \quad x^{1}=y^{0} \sinh y^{1}, \quad x^{2}=y^{2}, \quad x^{3}=y^{3} .
$$

Grant space is constructed by making topological identifications in the $y^{1}$ and $y^{2}$ directions:

$$
\left(y^{0}, y^{1}, y^{2}, y^{3}\right) \leftrightarrow\left(y^{0}, y^{1}+n a, y^{2}-n b, y^{3}\right)
$$

Misner space is the special case $b=0$. In Cartesian coordinates the above identification is equivalent to

$$
\begin{aligned}
\left(x^{0}, x^{1}, x^{2}, x^{3}\right) \leftrightarrow( & x^{0} \cosh (n a)+x^{1} \sinh (n a), \\
& \left.x^{0} \sinh (n a)+x^{1} \cosh (n a), x^{2}-n b, x^{3}\right) .
\end{aligned}
$$

It can be shown that Grant space is actually a portion of (holonomic to) Gott space, which consists of two infinitely long, straight cosmic strings passing by each other [10,17]. The periodicities $a$ and $b$ in Grant space are related respectively to the relative speed and distance between the two cosmic strings in Gott space. As $b$ approaches zero (the Misner space limit) the impact parameter of the two strings also approaches zero. 
Grant space can be considered as a portion of the $R^{2} \times T^{2}$ spacetime of the previous section with the boundaries in the $x^{1}$ direction moving toward each other at constant velocity. A spacetime diagram of the maximally extended Grant space is shown in Fig. 因. In regions I and IV radial straight lines represent $y^{1}=n a$ surfaces. Hyperbolas are constant $y^{0}$ surfaces. All image charges of a point are located on the same hyperbolic surface. As a particle crosses the radial boundary, $y^{1}=n a$, it is Lorentz boosted in a new inertial frame moving at a speed $v=\tanh a$ in the $x^{1}$ direction with respect to the original frame and is translated by $-b$ in the $x^{2}$ direction. What is extraordinary about Grant space is that it contains nonchronal regions (II and III). In those regions the roles of $y^{0}$ and $y^{1}$ are switched. The radial boundaries are now spacelike and the spacetime becomes periodic in the time $\left(y^{0}\right)$ direction. This topological identification allows the formation of CTC's in those regions. The boundaries $\left(x^{0}= \pm x^{1}\right)$ separating chronal regions (I and IV) and nonchronal regions (II and III) are chronology horizons which are a kind of Cauchy horizons. The chronological structure of Grant space is discussed in Ref. [17].

Within each interval between the periodic boundaries (i. e., one period) $\frac{\partial}{\partial x^{0}}$ can play the role of a timelike Killing vector field, but it is impossible to define a global timelike Killing vector field by patching these $\frac{\partial}{\partial x^{0}}$ 's together. Without a global timelike Killing vector field the vacuum state of the spacetime cannot be defined. However, we argue that the Minkowski vacuum state is a valid vacuum state of the Grant space. Each interval in Grant space is identical to a portion of Minkowski space, so a geodesic observer in the interval will not detect any particle in the Minkowski vacuum. Since the only difference between one interval to its neighbors is a constant relative velocity in the $x^{1}$ direction and a translation in the $x^{2}$ direction, geodesic observers in the neighboring intervals will not find any particles in the same vacuum state. The state in which no geodesic observer detects any particles can be considered as a vacuum state. This allows us to use the same renormalized Hadamard function Eq. (6) in the calculation of $\left\langle 0\left|T_{\mu \nu}\right| 0\right\rangle$ in Grant space.

The result of the calculation is most simply expressed in the Misner coordinates, $\left(y^{0}, y^{1}, y^{2}, y^{3}\right)$, 


$$
\begin{aligned}
\left\langle 0\left|T_{0}^{0}\right| 0\right\rangle= & \frac{M^{4}}{2 \pi^{2}} \sum_{n=1}^{\infty}\left[1+4 \xi \sinh ^{2}\left(\frac{n a}{2}\right)\right] \frac{K_{2}\left(z_{n}\right)}{z_{n}^{2}} \\
\left\langle 0\left|T_{1}^{1}\right| 0\right\rangle= & \frac{M^{4}}{2 \pi^{2}} \sum_{n=1}^{\infty}\left[1+4 \xi \sinh ^{2}\left(\frac{n a}{2}\right)\right] \\
& \times\left[\frac{K_{2}\left(z_{n}\right)}{z_{n}^{2}}-4 M^{2}\left(y^{0}\right)^{2} \sinh ^{2}\left(\frac{n a}{2}\right) \frac{K_{3}\left(z_{n}\right)}{z_{n}^{3}}\right] \\
\left\langle 0\left|T_{2}^{2}\right| 0\right\rangle= & \frac{M^{4}}{2 \pi^{2}} \sum_{n=1}^{\infty}\left\{\left[1+2(4 \xi-1) \sinh ^{2}\left(\frac{n a}{2}\right)\right] \frac{K_{2}\left(z_{n}\right)}{z_{n}^{2}}\right. \\
& \left.+M^{2}\left[4(1-4 \xi)\left(y^{0}\right)^{2} \sinh ^{4}\left(\frac{n a}{2}\right)-n^{2} b^{2}\right] \frac{K_{3}\left(z_{n}\right)}{z_{n}^{3}}\right\} \\
\left\langle 0\left|T_{3}^{3}\right| 0\right\rangle= & \left\langle 0\left|T_{22}\right| 0\right\rangle+\frac{M^{6} b^{2}}{2 \pi^{2}} \sum_{n=1}^{\infty} n^{2} \frac{K_{3}\left(z_{n}\right)}{z_{n}^{3}}
\end{aligned}
$$

where $z_{n}=M\left[4\left(y^{0}\right)^{2} \sinh ^{2}\left(\frac{n a}{2}\right)+n^{2} b^{2}\right]^{\frac{1}{2}}$. The trace is equal to

$$
\begin{aligned}
\left\langle 0\left|T_{\mu}^{\mu}\right| 0\right\rangle= & \frac{M^{4}}{2 \pi^{2}} \sum_{n=1}^{\infty}\left\{4\left[1+(6 \xi-1) \sinh ^{2}\left(\frac{n a}{2}\right)\right] \frac{K_{2}\left(z_{n}\right)}{z_{n}^{2}}\right. \\
& \left.+\left[-z_{n}^{2}+8(1-6 \xi) M^{2}\left(y^{0}\right)^{2} \sinh ^{4}\left(\frac{n a}{2}\right)\right] \frac{K_{3}\left(z_{n}\right)}{z_{n}^{3}}\right\}
\end{aligned}
$$

On the chronology horizon $\left(y^{0}=0\right)$, the components of the stress-energy tensor are

$$
\begin{aligned}
\left\langle 0\left|T_{0}^{0}\right| 0\right\rangle & =\frac{M^{4}}{2 \pi^{2}} \sum_{n=1}^{\infty}\left[1+4 \xi \sinh ^{2}\left(\frac{n a}{2}\right)\right] \frac{K_{2}(M n b)}{(M n b)^{2}} \\
\left\langle 0\left|T_{1}^{1}\right| 0\right\rangle & =\left\langle 0\left|T_{0}^{0}\right| 0\right\rangle \\
\left\langle 0\left|T_{2}^{2}\right| 0\right\rangle & =\frac{M^{4}}{2 \pi^{2}} \sum_{n=1}^{\infty}\left\{\left[1+2(4 \xi-1) \sinh ^{2}\left(\frac{n a}{2}\right)\right] \frac{K_{2}(M n b)}{(M n b)^{2}}-\frac{K_{3}(M n b)}{M n b}\right\} \\
\left\langle 0\left|T_{3}^{3}\right| 0\right\rangle & =\frac{M^{4}}{2 \pi^{2}} \sum_{n=1}^{\infty}\left[1+2(4 \xi-1) \sinh ^{2}\left(\frac{n a}{2}\right)\right] \frac{K_{2}(M n b)}{(M n b)^{2}}
\end{aligned}
$$

Figure 5 shows how the energy density $\rho$ depends on the field mass $M$ for the conformal coupling $\left(\xi=\frac{1}{6}\right)$. For $M<\frac{a}{b}, \rho$ diverges on the chronology horizon. The contribution to $\rho$ from the $n$th image charge for $n \gg 1$ is proportional to $\exp [n(a-M b)]$. The factor of $e^{n a}$ comes from the Doppler shift as the particle is boosted in the $y^{1}$ direction $n$ times. In Misner space this factor causes $\left\langle 0\left|T_{\mu \nu}\right| 0\right\rangle$ to diverge on the chronology horizon, and it might prevent the formation of CTC's. However, in Grant space the exponentially decaying factor $e^{-n M b}$, which comes from the nonvanishing geodesical distances between image charges in the $y^{2}$ direction $b$, makes $\left\langle 0\left|T_{\mu \nu}\right| 0\right\rangle$ finite for values of $M>\frac{a}{b}$. This result agrees with Boulware's 
similar calculation in Gott space [6]. This result implies that the metric backreaction may not be large enough to significantly change the Grant space geometry if the field mass is sufficiently large.

\section{DISCUSSION}

In calculating the vacuum expectation value of the stress-energy tensor for a massive scalar field with arbitrary curvature coupling in multiply connected flat spacetimes, we have shown that a nonzero field mass raises the value of the energy density (decreasing its magnitude) and confirmed that increased Möbiosity lowers the energy density. The introduction of nonorientability can evidently either increase the magnitude of the energy density (Möbius band) or decrease it (Klein bottle).

Another effect on the stress-energy that we have not examined yet is self-interaction (e. g., $\lambda \phi^{4}$ theory). The calculation of $\left\langle 0\left|T_{\mu \nu}\right| 0\right\rangle$ for a self-interacting field is much more complicated than that for a free field since it requires renormalization of quantities such as the field mass, which will be dependent on the field $\phi$, the curvature coupling constant $\xi$, and the field coupling constant $\lambda$. In spacetimes with relatively simple topology, such as $R^{3} \times T^{1}$ and Casimir-type spacetime, $\left\langle 0\left|T_{\mu \nu}\right| 0\right\rangle$ has been calculated by Birrell and Ford, Ford, Ford and Yoshimura, Kay, and Toms [18 23]. In a spacetime with CTC's self-interaction is known to cause failure of unitarity [24 28].

In Grant space we discovered that the divergence of $\left\langle 0\left|T_{\mu \nu}\right| 0\right\rangle$ on the chronology horizon depends on relative size of the field mass to the ratio of the periodicities. This result may have significant consequences for chronology protection. It suggests that the metric backreaction from the stress-energy of a massive quantized field will likely not be large enough to significantly alter the geometry and prevent the formation of CTC's. If quantized matter fields are to provide the chronology protection mechanism, our result would indicate that only massless fields may be capable of providing a sufficiently strong backreaction to prevent the formation of CTC's. Outside the domain of quantum gravity, this would place 
a heavy responsibility on the electromagnetic field (and conceivably neutrino fields, should any be massless) as the sole protector of chronology.

\section{ACKNOWLEDGMENTS}

This work was supported in part by National Science Foundation Grant No. PHY9207903. 


\section{REFERENCES}

* $\quad$ electronic mail address: tanaka@orion.oscs.montana.edu.

$\dagger \quad$ electronic mail address: billh@orion.oscs.montana.edu.

[1] B. S. DeWitt, C. F. Hart, and C. J. Isham, Physica 96A, 197 (1979).

[2] W. A. Hiscock and D. A. Konkowski, Phys. Rev. D 26, 1125 (1982).

[3] V. P. Frolov, Phys. Rev. D 43, 3878 (1991).

[4] S.W. Kim and K. S. Thorne, Phys. Rev. D 43, 3929 (1991).

[5] G. Klinkhammer, Phys. Rev. D 46, 3388 (1992).

[6] D. G. Boulware, Phys. Rev. D 46, 4421 (1992).

[7] J. D. E. Grant, Phys. Rev. D 47, 2388 (1993).

[8] M. Lyutikov, Phys. Rev. D 49, 4041 (1994).

[9] T. Tanaka and W. A. Hiscock, Phys. Rev. D 49, 5240 (1994).

[10] D. Laurence, Phys. Rev. D. 50, 4957 (1994).

[11] N. D. Birrell and P. C. W. Davies, Quantum fields in curved space (Cambridge University Press, Cambridge, England, 1982), pp. 87.

[12] S. A. Fulling, Aspects of Quantum Field Theory in Curved Spacetime (Cambridge University, Cambridge, England, 1989), pp. 85.

[13] R. M. Wald, General Relativity (The University of Chicago Press, Chicago, USA, 1984), pp. 363.

[14] S. W. Hawking, Phys. Rev. D 46, 603 (1992).

[15] C. W. Misner, in Relativity Theory and Astrophysics I: Relativity and Cosmology, Lectures in Applied Mathematics, edited by J. Ehlers (American Mathematical Society, 
Providence, 1967), pp. 160-169.

[16] S. W. Hawking and G. F. R. Ellis, The Large Scale Structure of Space-time (Cambridge University, Cambridge, England, 1973), pp. 170-174.

[17] For a recent review of these notions, see K. S. Thorne, in General Relativity and Gravitation 1992, Proceeding of the Thirteenth International Conference on General Relativity and Gravitation, edited by R. J. Gleiser, C. N. Kozameh, and O. M. Moreschi (Institute of Physics, Bristol, 1993), pp. 295.

[18] L. H. Ford, Proc. R. Soc. Lond. A 368, 305 (1979).

[19] L. H. Ford and Tetz Yoshimura, Phys. Lett. 70A, 89 (1979).

[20] B. S. Kay, Phys. Rev. D 20, 3052 (1979).

[21] N. D. Birrell and L. H. Ford, Phys. Rev. D 22, 330 (1980).

[22] D. J. Toms, Ann. Phys. 129, 334 (1980).

[23] D. J. Toms, Phys. Rev. D 21, 928 (1980).

[24] J. Friedman, M. S. Morris, I. D. Novikov, F. Echeverria, G. Klinkhammer, K. S. Thorne, and U. Yurtsever, Phys. Rev. D 42, 1915 (1990).

[25] F. Echeverria, G. Klinkhammer, and K. S. Thorne, Phys. Rev. D 44, 1077 (1991).

[26] I. D. Novikov, Phys. Rev. D 45, 1989 (1992).

[27] J. L. Friedman, N. J. Papastamatiou, and J. Z. Simon, Phys. Rev. D 46, 4442 (1992).

[28] J. L. Friedman, N. J. Papastamatiou, and J. Z. Simon, Phys. Rev. D 46, 4456 (1992).

[29] M. S. Morris, K. S. Thorne, and U. Yurtsever, Phys. Rev. Lett. 61, 1446 (1988). 


\section{FIGURES}

FIG. 1. Plots of the energy density $\rho=\left\langle 0\left|T_{00}\right| 0\right\rangle$ vs. the field mass $M$ for orientable manifolds for a conformally coupled field $\left(\xi=\frac{1}{6}\right)$ with periodicities $a=b=c=1$. From top to bottom the curves represent the topologies $R^{3} \times S^{1}, R^{2} \times T^{2}$, and $R^{1} \times T^{3}$. Increasing the field mass decreases the magnitude of the energy density while increasing Möbiosity increases it.

FIG. 2. Plots of the energy density $\rho=\left\langle 0\left|T_{00}\right| 0\right\rangle$ vs. the field mass $M$ for the Möbius strip and Klein bottle at $x^{2}=0$ for a conformally coupled field $\left(\xi=\frac{1}{6}\right)$. The periodicities $(a$ and $b)$ are set to 1 . From top to bottom the curves represent topologies $R^{3} \times S^{1}$, the Möbius strip,the Klein bottle, and $R^{2} \times T^{2}$.

FIG. 3. Plots of the energy density $\rho=\left\langle 0\left|T_{00}\right| 0\right\rangle$ vs. $x^{2}$ coordinate in the Möbius strip (solid line) and Klein bottle (dashed line) with $\xi=\frac{1}{6}$ and $a=b=M=1 . \rho$ decays quickly as we move away from $x^{2}=0$ on the Möbius strip whereas it is periodic on the Klein bottle.

FIG. 4. Spacetime diagram of the maximally extended Grant space. The radial straight lines are identified periodic boundaries at $y^{1}=n a$, and the hyperbolas are surfaces of constant $y^{0}$ in regions I and IV. Points A and B are identified with each other. As a particle crosses the boundary from one interval to next, it is Lorentz boosted in the $x^{1}$ direction and translated in the $x^{2}$ direction by $-b$. The roles of $x^{0}$ and $x^{1}$ are switched in nonchronal regions II and III. Two images charges $\mathrm{C}$ and $\mathrm{D}$ can be connected by a timelike curve. CTC's exist in these regions.

FIG. 5. The energy density of a massive conformal scalar field on the chronology horizon in Grant space. The periodicities $a$ and $b$ are both set to 1 . In the shaded region $\left(M<\frac{a}{b}=1\right) \rho$ diverges and possibly prevents formation of CTC's by back reaction on spacetime geometry. For $M>\frac{a}{b}, \rho$ remains finite on the chronology horizon. At the critical value $M=\frac{a}{b}=1$, the limiting value of $\rho$ is equal to -0.106 . 
Figure 1

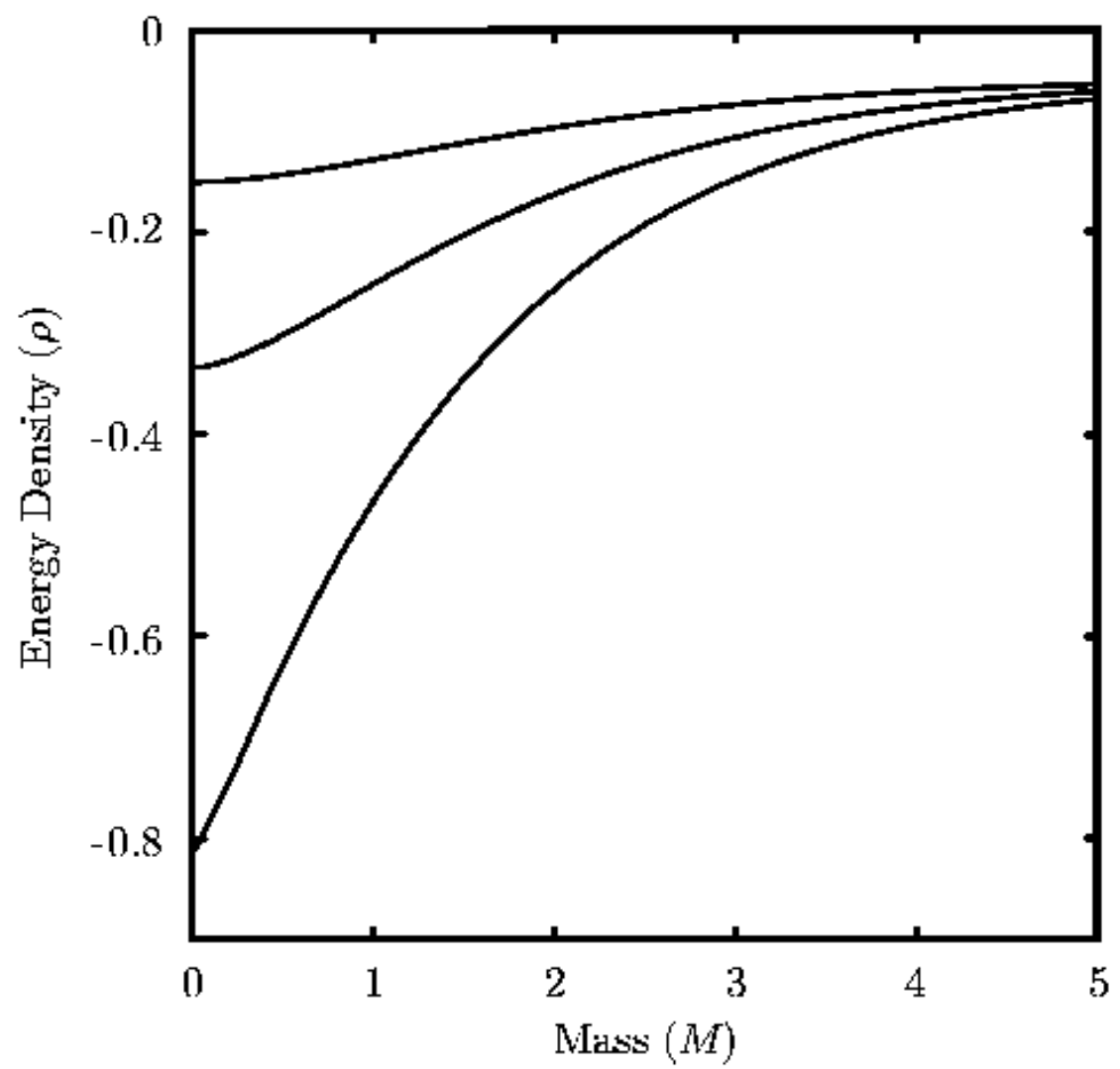


Figure 2

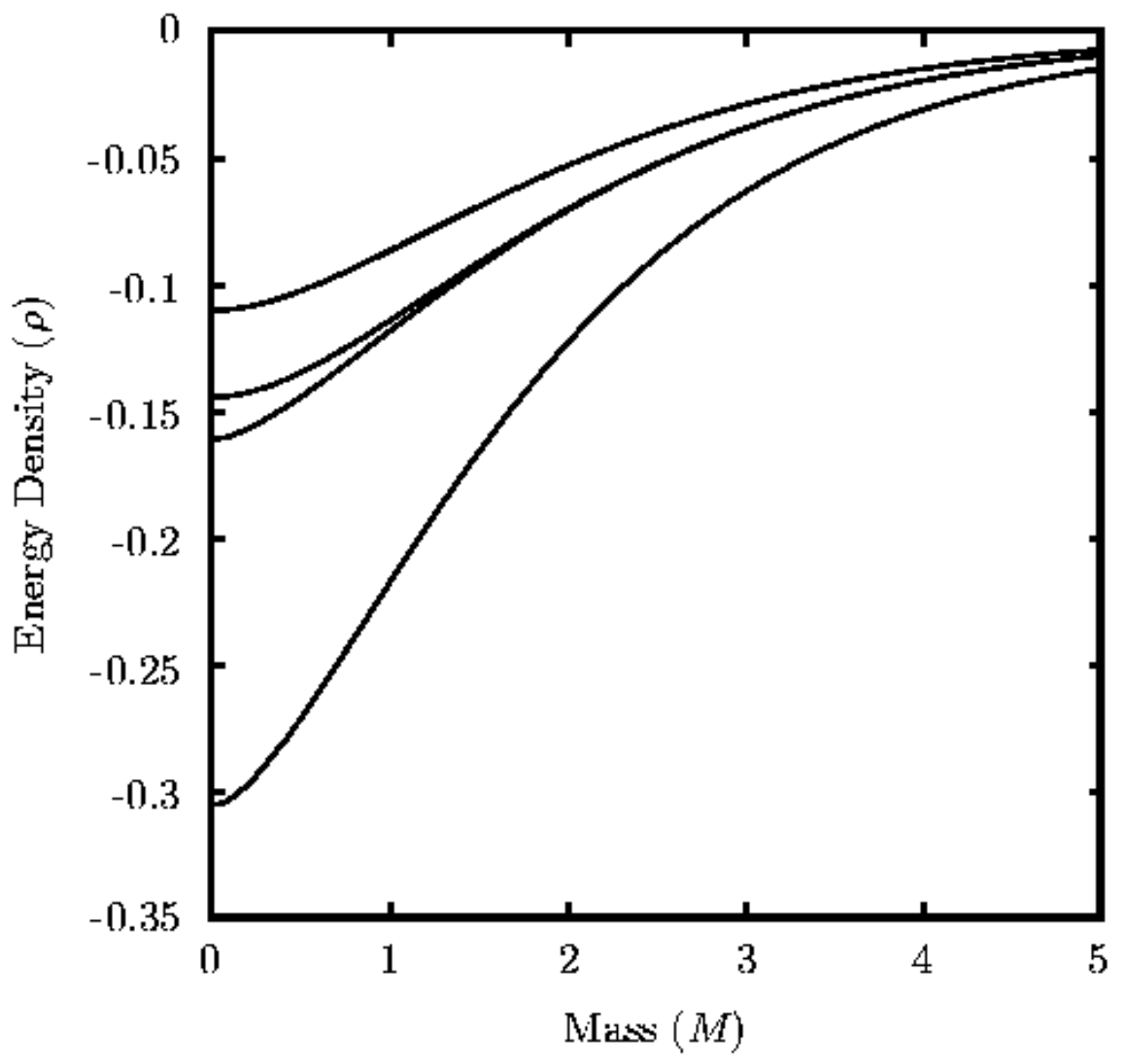


Figure 3

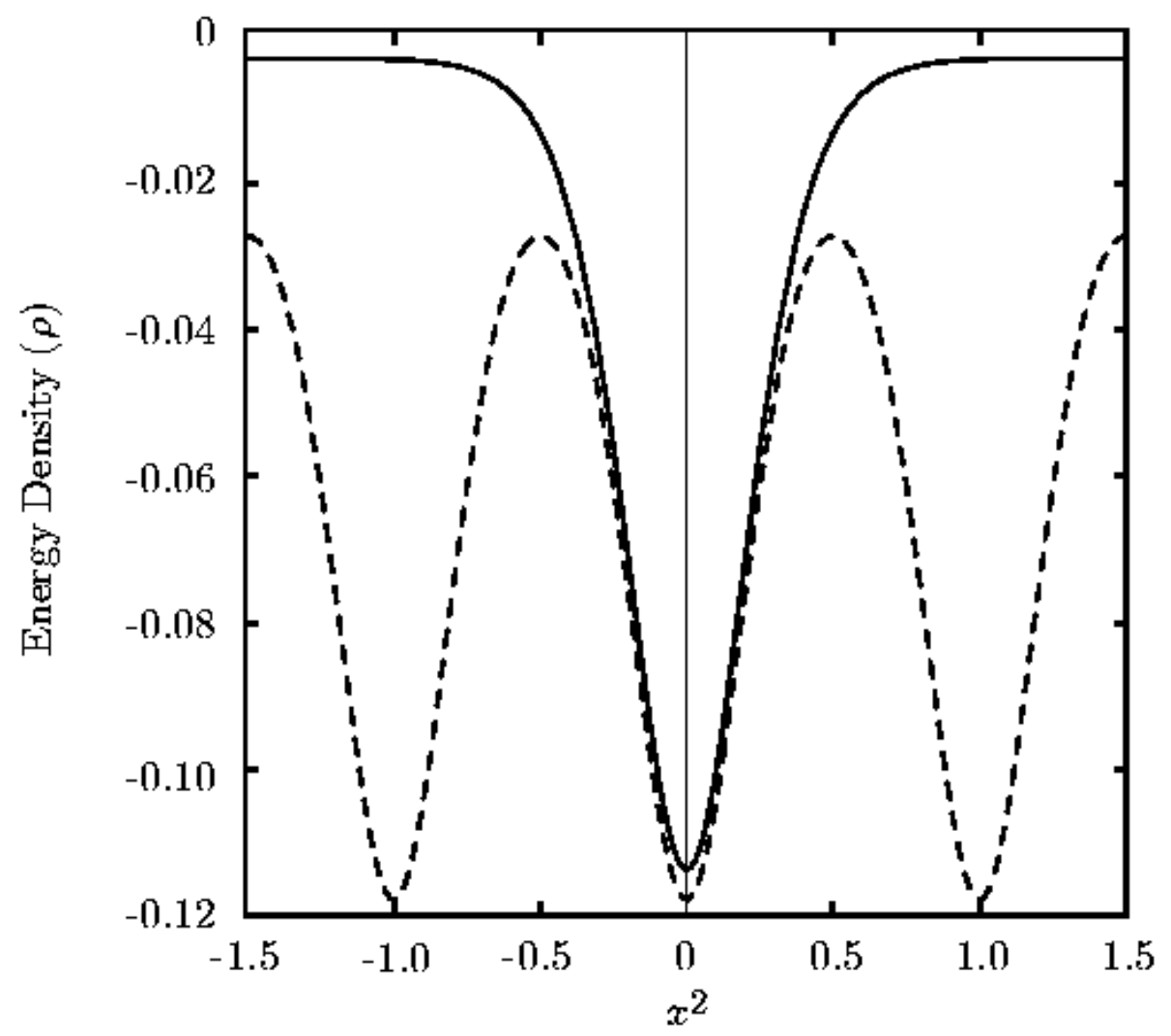


Figure 4

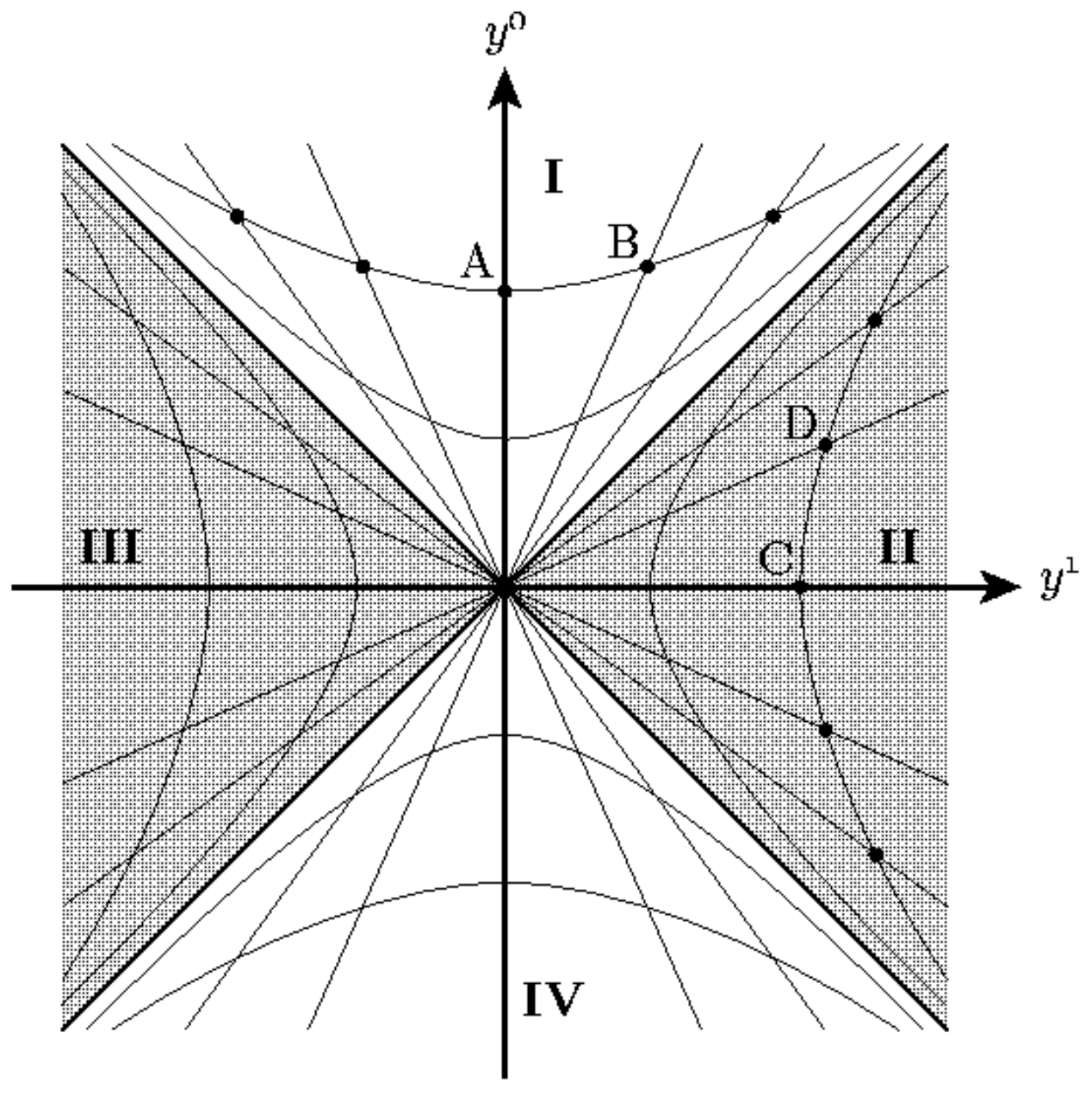




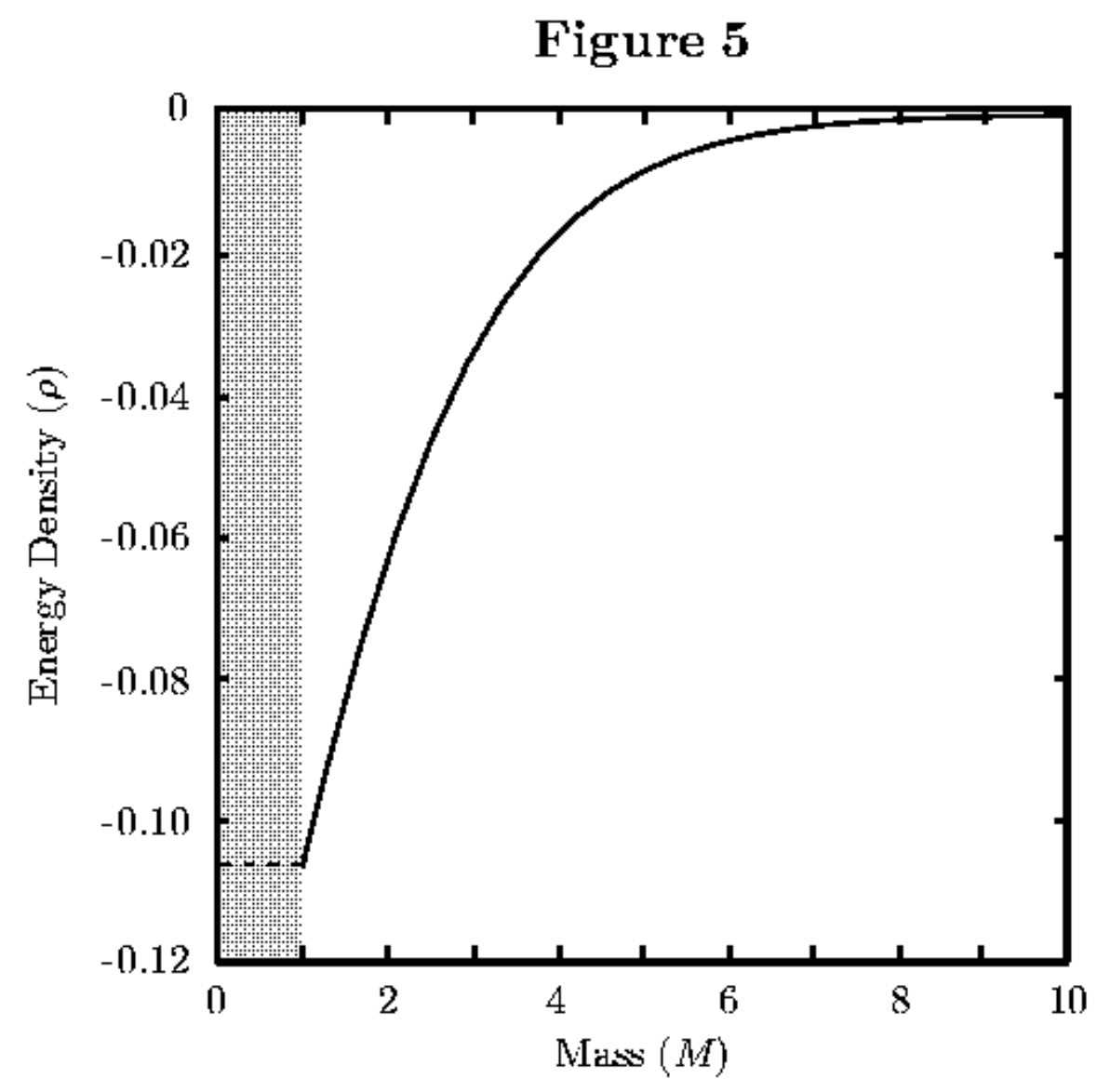

